\title{
Overexpression of KLF14 protects against immune-mediated hepatic injury in mice
}

\author{
Xiaoyan Chen ${ }^{1} \cdot$ Qinghai Tan ${ }^{1,2} \cdot$ Yunwu Wang ${ }^{1} \cdot$ Huijie Lv $^{1} \cdot$ Zhihui Wang $^{1} \cdot$ Zhuoying Lin $^{1} \cdot$ Zhipeng Du$^{1} \cdot$ \\ Si Xiong ${ }^{1} \cdot$ Jian Han $^{1} \cdot$ Dean Tian ${ }^{1} \cdot$ Bo Wang ${ }^{1}$
}

Received: 15 February 2018 / Revised: 26 July 2018 / Accepted: 27 July 2018 / Published online: 25 September 2018

(c) United States \& Canadian Academy of Pathology 2018

\begin{abstract}
The underlying immunopathogenic mechanisms of autoimmune hepatitis (AIH) have not yet been well elucidated. An impairment in regulatory T cells (Tregs) is key to the development of AIH. Krüppel-like factors (KLFs) regulate a broad of cellular processes including immunocyte maturation. KLF14 may regulate Treg differentiation, but the biological functions remain far from elucidated. In this study, we identified the hepatic expression of KLF14 in human and murine liver diseases. Immune-mediated hepatitis was induced by concanavalin A (Con A). A KLF14 recombinant adenoviruses plasmid (AdKLF14) was constructed and injected into mice. Tregs were assessed by flow cytometry analysis; inflammatory cytokines, such as tumor necrosis factor $\alpha(\mathrm{TNF}-\alpha)$ and interleukin 6 (IL-6), were tested by enzyme-linked immunosorbent assay (ELISA). The expression of KLF14 was suppressed in a time-and dose-dependent manner. Changes in cytokine levels were consistent with the degree of hepatic injury. Overexpression of KLF14 protected the liver from immune-mediated damage in vivo. Ad-KLF14 transfection before Con A challenge increased the frequency of Tregs in liver mononuclear cells (MNCs), and suppressed the expression of cytokines. All of these improvements were completely abrogated after Treg deletion in vivo by intraperitoneal injection of a CD25 antibody. In conclusion, these data suggest that KLF14 plays an asyet unrecognized role in immune-mediated hepatitis mainly via induced Treg differentiation. Our findings extend the knowledge of the biological function of KLF14 to the autoimmune disease field, and indicate the possibility of KLF14 as a therapeutic target in AIH patients.
\end{abstract}

\section{Introduction}

Autoimmune hepatitis (AIH) represents a chronic clinical entity characterized by hypergammaglobulinemia, circulating autoantibodies, and interface hepatitis [1]. The disease tends to lead to cirrhosis, liver failure, and even death. Most patients, if managed in time, respond well to

Electronic supplementary material The online version of this article (https://doi.org/10.1038/s41374-018-0134-4) contains supplementary material, which is available to authorized users.

Bo Wang

wangbo@tjh.tjmu.edu.cn

1 Department of Gastroenterology, Tongji Hospital, Tongji Medical College, Huazhong University of Science and Technology, Wuhan, China

2 Department of Gastroenterology, Hannover Medical School, Hannover, Germany immunosuppression. For those patients not responsive to steroids or azathioprine, second-line therapies can prevent progression of the disease. Mycophenolate mofetil, rapamycin, and infliximab have been shown to achieve biochemical improvements and to be promising treatment alternatives for hepatitis [2].

The development of AIH entails an intricate interaction between genetic and environmental factors. Susceptibility to $\mathrm{AIH}$ is associated with some specific alleles within the major histocompatibility complex (MHC) on the short arm of chromosome 6 [3]. Moreover, the polymorphisms of inflammatory cytokine genes, such as TNF- $\alpha$, also contribute to the pathogenesis of AIH [4]. It has been generally accepted that the imbalance between regulatory and effector factors results in the breakdown of immune tolerance and development of autoimmune diseases [5]. Since class II MHC molecules control the presentation of antigen to $\mathrm{CD}^{+} \mathrm{T}$ lymphocytes, activated $\mathrm{T}$ cells are, presumably, involved in the pathogenesis of AIH. A subpopulation of $\mathrm{CD}^{+} \mathrm{T}$ lymphocytes, namely $\mathrm{CD} 4^{+} \mathrm{CD} 25^{+} \mathrm{Foxp}^{+}$ 
regulatory $\mathrm{T}$ cells (Tregs) has been found to be intimately associated with human autoimmune conditions. Studies over the past few decades have demonstrated that impairment in Tregs, in terms of number and function, is crucial to the development of AIH [6, 7].

Nonetheless, the immunopathogenic mechanisms of AIH remain poorly understood. One of the major problems with the study on the development of AIH is the lack of reliable animal models. In this study, we employed intravenous injection of Concanavalin A (Con A) to create an immunemediated liver inflammatory model in mice. This extensively used model can partially mimic the initial phase of human T cells-mediated liver diseases, such as AIH and viral hepatitis and can explicitly show the involvement of $\mathrm{CD} 4{ }^{+} \mathrm{T}$ cells in the induction and progression of hepatic injury $[8,9]$.

Krüppel-like factors (KLFs) belong to the evolutionarily conserved family of zinc finger-containing transcription factors. It has been well-established that these genes regulate a wide array of cellular processes, such as proliferation, differentiation, metabolism, and apoptosis. To date, the KLFs family consists of 17 identified members and three distinct groups based on their functional features [10]. Family members in Group 2 (KLFs 1, 2, 4, 5, 6, and 7) serve as transcriptional activators, while the other are repressors, which inhibit transcription by interacting with the C-terminal binding protein (Group 1, KLFs 3, 8, and 12) or Sin3A (Group 3, other KLFs), except KLF14. Its biological functions remain far from being understood.

Multiple studies have indicated that KLFs are pathophysiologically implicated in the development of liver diseases. For instance, KLF2 and KLF11 play some roles in the hepatic fibrogenesis. Moreover, KLF4, 6, 8, 9, 10, and 17 have been shown to be involved in the pathogenesis of hepatocellular carcinoma (HCC) by modulating the invasion, proliferation, apoptosis, and migration [11].

Despite recent advances, the function of KLF proteins in the immune system remain elusive. Several KLFs, such as KLF 2, 4, 6 play essential roles in the cellular maturation of $\mathrm{T}$ cells, B cells, Th17 and macrophages, etc [12]. KLF 2, 10, and 14 can control the biological behavior of Tregs [13, 14]. KLF14 was able to directly modulate Treg differentiation via chromatin remodeling at the Treg-specific demethylation region in forkhead transcription factor $\mathrm{p} 3$ (Foxp3) gene [15]. Nevertheless, no studies explored the role of KLF14 in immune-mediated hepatitis, and in this study, our research effort was mainly directed at KLF14.

To gain insight into the role of KLF14 in immunemediated hepatitis, we examined the expressions of KLF14 mRNA and protein under the Con A stimulation. Our results indicate that overexpression of KLF14 can alleviate the hepatic injury induced by Con A. This protection might be associated with the differentiation of Tregs, as well as inhibition of the expression of some inflammatory cytokines, such as TNF- $\alpha$ and interleukin-6 (IL-6). Taken together, these findings expand our knowledge about the biological function of KLF14 in autoimmune diseases, including its protective effect on immune-mediated hepatitis.

\section{Materials and methods}

\section{Antibodies}

The following antibodies were used: CD25 antibody (clone PC-61.5) and isotype-control rat $\operatorname{IgG}$ (clone HRPN) for neutralization assay were obtained from BioXcell (West Lebanon, NH); KLF14 (SAB1304202) and $\beta$-actin (A1978) antibody were procured from Sigma for western blotting; TNF- $\alpha$ antibody (3707) for co-immunoprecipitation (co-IP) were purchased from Cell Signaling Technology; anti-CD4FITC and anti-CD25-APC antibodies for Flow cytometry analysis were bought from eBioscience (San Diego, USA).

\section{Animals}

Male C57BL/6 wild-type mice aged 7-8 weeks were maintained under pathogen-free conditions and kept in an animal facility at Tongji Medical College, Wuhan, China. All procedures in this study were carried out in accordance with the criteria outlined in the "Guides for the Care and Use of Laboratory Animals" by the National Academy of Sciences, China.

\section{Disease models}

Immune-mediated hepatitis was induced by intravenous injection at a single dose of freshly-prepared Con A (15 mg/ kg, Sigma-Aldrich Chemical Co., St. Louis, MO), reconstituted in sterile phosphate-buffered saline (PBS) $(n=8)$. After Con A administration, blood samples were collected at different time points for measurement of plasma alanine transaminase (ALT) and aspartate aminotransferase (AST) levels (Biotron Diagnostics, Hemet, CA). Livers were then perfused with ice-cold PBS to remove blood components. Liver sections were processed and stained with hematoxylin and eosin (H\&E) according to standard protocols for histological evaluation of the liver injury. For Immunohistochemistry, antibodies against KLF14 (Santa Cruze, USA) were applied to paraffin-embedded liver sections according to the standard protocols.

Other models of hepatic disease were made as previously described [16, 17]. In brief, mouse-model of nonalcoholic fatty liver disease (NAFLD) was established by feeding mice with a high-fat diet (HFD; 53\% of calories from fat) 
for 12 weeks $(n=8)$, and liver fibrosis model was induced by intraperitoneally injecting $20 \%$ carbon tetrachloride (CCL4; $0.5 \mu \mathrm{l} / \mathrm{g}$ of body weight twice a week) for 8 weeks ( $n=8)$. Following overnight-fasting, the mice were sacrificed, and liver tissue samples were snap-frozen in liquid nitrogen for RNA extraction.

\section{Clinical specimens}

A total of 15 pairs of hepatocellular carcinoma (HCC) and matched non-tumor samples were obtained, with informed consent, from patients who had undergone radical resection at the Department of Hepatic Surgery, Tongji Hospital, Wuhan, China. The tissue was acquired upon the approval of the Ethics Committee of the Tongji Hospital and according to the ethical standards of the World Medical Association Declaration of Helsinki. Samples were flashfrozen and stored in liquid nitrogen before RNA extraction.

\section{Cell isolation and culture}

Primary mouse hepatocytes (HCs) and liver mononuclear cells (MNCs) were isolated as previously described [18]. In brief, mice livers were perfused with liberase TM solution (Roche, Mannheim, Germany) after anesthesia, filtered with $70 \mu \mathrm{m}$ nylon cell strainer (BD Falcon) and centrifuged at $20 \times g$ for $5 \mathrm{~min}$. The pellets (HCs) were suspended and put onto the surface of $30 \%$ Percoll solution, centrifuged at $1000 \times g$ for $10 \mathrm{~min}$ at $4{ }^{\circ} \mathrm{C}$ and washed once with PBS. Supernatants containing MNC were collected, resuspended in $30 \%$ Percoll and gently overlaid onto $70 \%$ Percoll. After centrifugation at $1000 \times g$ for $30 \mathrm{~min}$, liver MNCs were harvested from the interphase, washed twice with PBS, and then resuspended for further fluorescence activated cell sorting (FACS) analysis.

Peripheral blood mononuclear cells (PBMC) were collected from mice blood samples by centrifugation at $400 \times g$ for $20 \mathrm{~min}$ by using mononuclear cell-separating solution (Tbdscience, Tianjin, China) according to the standard protocols.

Spleen was ground and filtered with $70 \mu \mathrm{m}$ nylon cell strainer (BD Falcon) in PBS. After erythrocytes were depleted, the purified splenocytes were centrifuged and taken for further flow cytometry.

$\mathrm{CD} 4{ }^{+} \mathrm{CD} 25^{+}$regulatory $\mathrm{T}$ cells were prepared by depleting non-CD4 ${ }^{+} \mathrm{T}$ cells from splenic and lymph node cells by employing magnetic cell sorting (MACS, Miltenyi, Bergisch Gladbach, Germany) and then by positive selection of $\mathrm{CD}_{25}{ }^{+}$cells using anti-CD25-PE plus anti-PE beads. The sorted cells (purity $>95 \%$ ) were collected and isolated RNA for quantitative real-time PCR.

Mouse macrophage RAW264.7 cell line (RAW264.7) was cultured in Dulbecco's modified Eagle's medium supplemented with $10 \%$ heat-inactivated fetal bovine serum at $37^{\circ} \mathrm{C}$ in $5 \% \mathrm{CO}_{2}$ atmosphere.

\section{Plasmid transfection}

Mouse KLF14 (CMV-MCS-3FLAG-SV40-Neomycin) plasmid was purchased from Genechem (Shanghai, China). Recombinant adenoviruses (Ad-KLF14), expressing mouse KLF14 and driven by the cytomegalovirus immediate early gene promoter, were packaged by GeneChem (Shanghai, China). Briefly, recombinant plasmids were transfected into 293 cells to obtain adenovirus stocks, then purified by double cesium chloride gradient ultracentrifugation. Viral titer was determined by plaque assay and expressed in plaque forming units (pfu). Purified aliquots were stored at $-80^{\circ} \mathrm{C}$.

The RAW264.7 cells were transfected with mouse plasmids using Lipofectamine 3000 reagent (Carlsbad, CA, USA). The efficiency was assessed by real-time PCR and western blotting.

To enhance KLF14 response in the liver, we pretreated mice with recombinant adenoviruses plasmid (Ad-KLF14) $72 \mathrm{~h}$ before Con A stimulation. A plasmid solution containing $1 \times 10^{9}$ plaque-forming units of KLF14 per mouse was injected via tail vein. For negative control, null (Adnegative) type 5 adenovirus was injected.

\section{Neutralization assay}

In vivo depletion of Tregs was achieved by intraperitoneal injection of $250 \mu \mathrm{g}$ anti-CD25 Mab or isotype-control rat IgG. All groups $(n=5-8)$ received Con A challenge $24 \mathrm{~h}$ after antibody injection.

\section{RNA extraction and quantitative real-time PCR (qRT- PCR)}

Total RNA was extracted using TRIzol reagent and transcribed into cDNA using the PrimeScript RT reagent kit (Takara, Otsu, Japan). Real-time polymerase chain reaction was performed using SYBR Premix ExTaq (Takara, Otsu, Japan) on an ABI StepOne Real-Time PCR System (Applied Biosystem, Carlsbad, CA, USA). The experiments were repeated in triplicates, and the relative expression levels were determined by the $2^{-\Delta \Delta \mathrm{Ct}}$ method. The sequences of the primers used for PCR were listed in Table 1 in Supplementary Information.

\section{Western blotting and co-immunoprecipitation (Co-IP)}

Total proteins were extracted and subjected to Western blot analysis. Antibodies against $\beta$-actin and KLF14 were used. The membrane was incubated overnight at $4{ }^{\circ} \mathrm{C}$ with primary antibodies, and then washed three times in TBS 
containing $0.1 \%(\mathrm{v} / \mathrm{v})$ Tween 20 or BSA for 15 min prior to incubation with horseradish peroxidase (HRP) anti-rabbit $\operatorname{IgG}$ (1:2000; Sigma, CA), or HRP anti-mouse $\operatorname{IgG}$ (1:2000; Santa Cruz Inc., USA). The signals were detected with enhanced chemiluminescence (Thermo Fisher, Carlsbad, CA, USA).

For co-immunoprecipitation, RAW264.7 cells were transfected with KLF14 plasmids and then lysed with $1 \%$ NP40 NET buffer (Promoter Company, China). The supernatants were incubated overnight at $4{ }^{\circ} \mathrm{C}$ with protein G-Sepharose beads (Sigma) conjugated with rabbit antiFlag. The beads were then washed four times with lysis buffer, and the precipitates were eluted with sample buffer, separated by SDS/PAGE, and analyzed by immunoblotting.

\section{Enzyme-linked immunosorbent assay (ELISA)}

Serum from mice was harvested and tested for the expression of TNF- $\alpha$ and IL- 6 protein by following the instructions of the manufacturer (R\&D Systems, China).

\section{Flow cytometry}

Isolated mouse PBMC, liver MNC, and splenocytes were resuspended in PBS containing 1\% BSA, and stained with anti-CD4-FITC and anti-CD25-APC antibodies for $30 \mathrm{~min}$ at $4{ }^{\circ} \mathrm{C}$ in the dark. Tregs were stained by using mouse regulatory $\mathrm{T}$ cell staining kit (eBioscience, San Diego, USA). Data were analyzed by using a FlowJo software package (TreeStar, Ashland, OR, USA).

\section{Statistical analysis}

Data are expressed as mean \pm standard error. Each experiment was done in triplicate unless otherwise noted, and results were analyzed by using Student's t-test. Statistical analysis was performed by employing Prism 5.0 (GraphPad Software, La Jolla, CA, USA). A $p<0.05$ was considered to be statistically significant.

\section{Results}

\section{KLF expression in liver tissue with Con A-induced hepatitis}

We first characterized the time course of hepatic injury after Con A injection. As shown in Fig. 1a, $6 \mathrm{~h}$ after Con A injection, grossly, there was liver congestion (Fig. 1a upper), and microscopically, there were slight degenerative changes of hepatocytes (Fig. 1a middle), but no necrosis and cellular infiltration were noted. After $12 \mathrm{~h}$, spotty hepatocellular necrosis, sinusoidal hyperemia associated with mononuclear cell infiltration were found. Twenty-four hours after the injection, focal hepatocellular necrosis developed, and extensive infiltration of mononuclear cells were seen in the portal area within the Glisson's capsules and around the central vein (Fig. 1a lower). These changes remained $48 \mathrm{~h}$ after Con A injection. All mice receiving Con $\mathrm{A}$ at $15 \mathrm{mg} / \mathrm{kg}$ body weight survived.

Plasma transaminase activity was detected at the corresponding time points after Con A injection. The detection showed that Con A elicited a striking increase in plasma transaminase level. Such an increase was detectable as early as $6 \mathrm{~h}$ after Con A injection, and after reaching the peak levels at around $12 \mathrm{~h}$, the level dropped within $48 \mathrm{~h}$ (Fig. 1b).

TNF- $\alpha$ and IL- 6 are, in general, two most representative inflammatory cytokines in AIH patients. In this study, we detected the expression of TNF- $\alpha$ and IL-6 in mice serum by using ELISA. Our data indicated that these cytokines were significantly upregulated, with the peak response occurring roughly $6 \mathrm{~h}$ after the injection (Fig. 1c).

\section{KLF14 expression is downregulated in chronic liver diseases}

Little is known about the role of KLF14 in chronic hepatic damage. To determine whether the downregulation of KLF14 is transient or the result of Con A stimulation, we examined the expression of KLF14 at the transcriptional level in mice and patients with chronic liver diseases. As shown in Fig. 2a, b, the KLF14 was inhibited at least in mice with liver fibrosis (induced by CCL4 for 8 weeks), in mice with NFALD (induced by HFD for 12 weeks), and in human subjects with liver cancer. Our results indicated that KLF14 level might be negatively associated with the severity of the chronic hepatic injury.

To kinetically characterize the suppression of KLF14 with Con A, mice $(n=5)$ were injected intravenously at a single dose of $15 \mathrm{mg} / \mathrm{kg}$ Con A. liver samples were harvested 6, 12, 24, and $48 \mathrm{~h}$ after injection. KLF14 RNA level was detected by qRT-PCR (Fig. 2c) and the protein level was measured by western blotting (Fig. 2d). Our data showed that Con A changed the KLF14 expression in a time-dependent manner. Gradient concentrations of Con A (from 5 to $20 \mathrm{mg} / \mathrm{kg}$ ) were injected for $24 \mathrm{~h}(n=5)$. The result indicated that KLF 14 expression was suppressed by Con A (Fig. 2e), and the plasma transferase level was elevated, both in a dose-dependent manner (Fig. 2f).

To investigate the KLF14 expression within the liver, paraffin-embedded liver specimens were immunohistochemically examined. Figure $2 \mathrm{~g}$ shows that the nuclei of hepatocytes and non-parenchymal cells were positively stained. Con A treatment reduced the expression of KLF14 proteins in these cells. We verified these results at the 
A
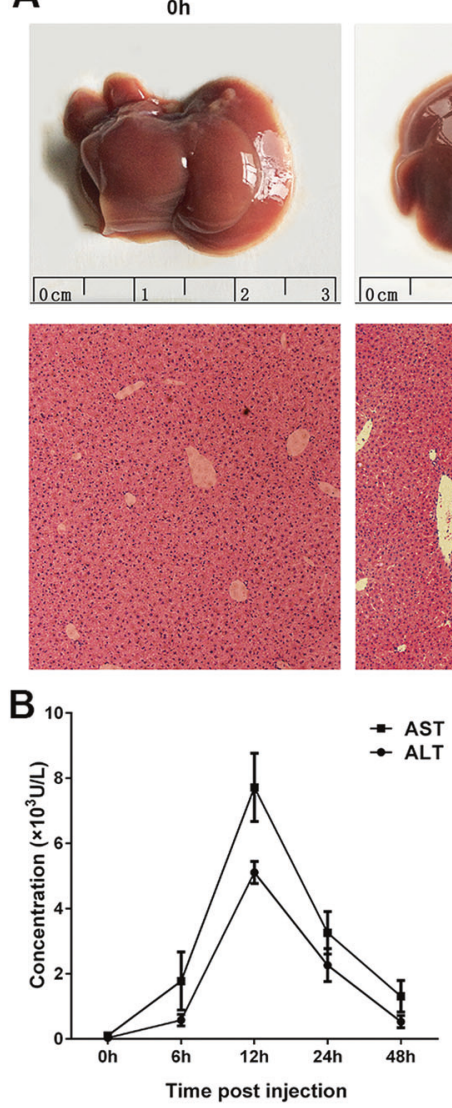

$6 h$
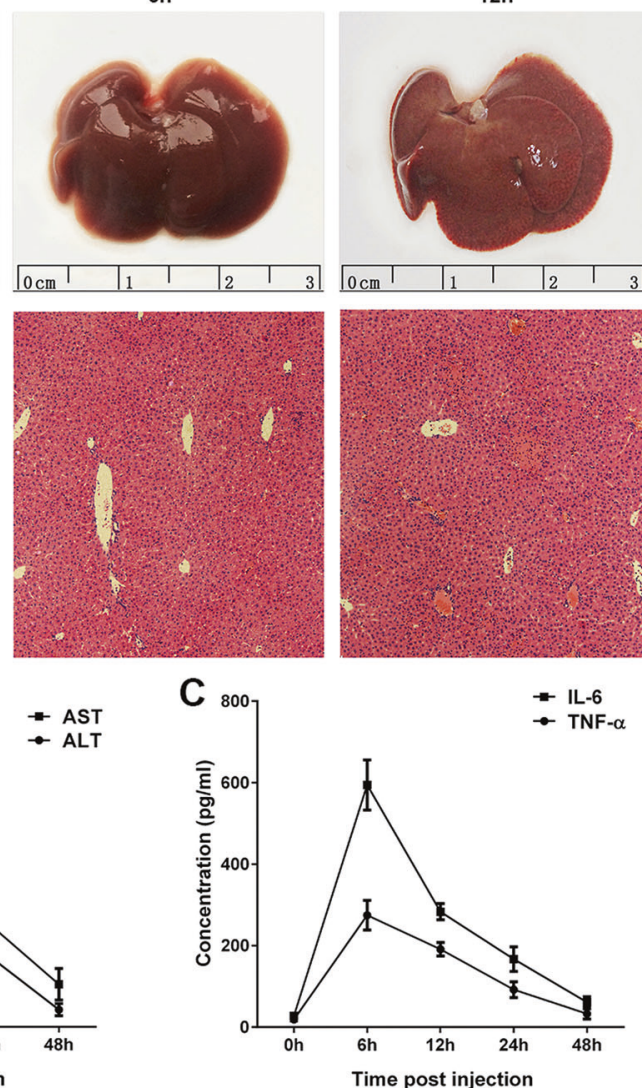

$12 \mathrm{~h}$
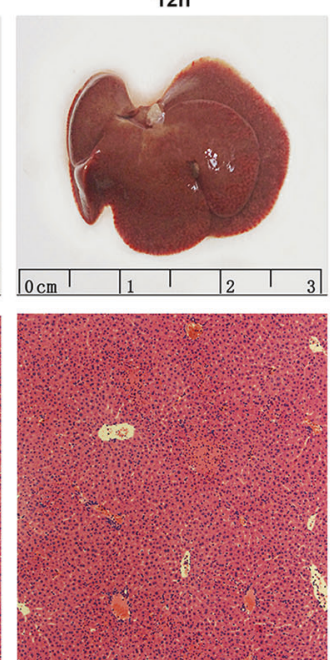

Time post injection
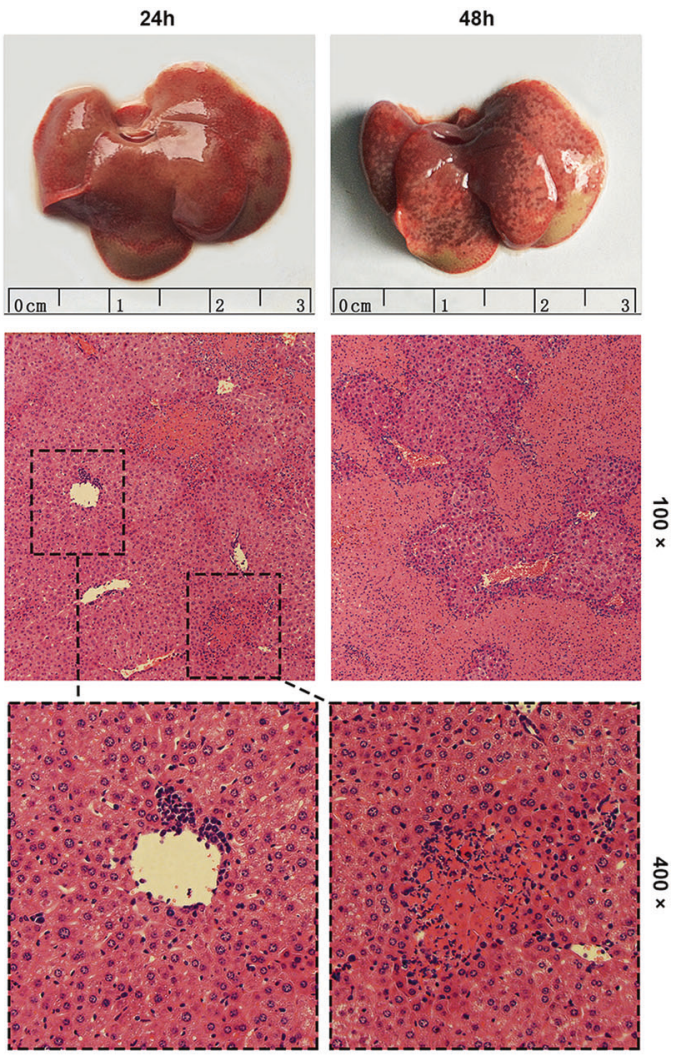

Fig. 1 KLF expression in liver tissue with Con A-induced hepatitis. a Liver samples (upper) and histological findings (middle, H\&E staining, magnification $\times 100$ ) from mice sacrificed at different time points after intravenous injection of Con A $(15 \mathrm{mg} / \mathrm{kg})$ into C57BL/6 mice aged 7-8 weeks. Representative results $24 \mathrm{~h}$ after injection are shown at high power (lower, magnifications $\times 400$ ). b Serum transaminase

concentrations (ALT and AST) were determined at corresponding time points after Con A injection. $\mathbf{c}$ Serum from mice at corresponding time points after Con A injection was collected and tested for the expression of TNF- $\alpha$ and IL- 6 protein by ELISA. Data are expressed as mean \pm SD

cellular level (Fig. 2h). Proteins were isolated from HCs and MNC $24 \mathrm{~h}$ after Con A injection. Western blotting showed that, compared to the control group (PBS injection), the KLF14 expression was downregulated.

\section{The protective effect of KLF 14 overexpression on immune-mediated damage}

On the basis of the aforementioned results, we were led to theorize that KLF 14 might play a role in Con A-induced liver damage. To further elucidate the role, recombinant adenoviruses plasmid (Ad-KLF14) was constructed and injected into the mice. qRT-PCR and Western blotting demonstrated that the KLF14 was upregulated in the liver tissue (data are presented in Supplemental Fig. 1). Mice were then challenged with Con A $72 \mathrm{~h}$ later. Our result showed that, compared with negative control group (Adnegative mice), KLF 14 overexpression in liver alleviated the Con A-induced hepatic damage, and histological examination revealed only spotty necrosis and mild mononuclear infiltration (Fig. 3a). We also found that plasma transferase level was decreased (Fig. 3b). To study the molecular mechanism of this KLF14-related improvement, we measured the TNF- $\alpha$ and IL- 6 expressions by using ELISA. Fig. 3c shows that pretreatment with AdKLF14 down-regulated the expression of these inflammatory cytokines.

We then transfected RAW264.7 cells with mouse plasmid encoding KLF14-Flag to determine whether KLF14 could work together with TNF- $\alpha$ by co-immunoprecipitation. The expression of KLF14 in the cell line was up-regulated at RNA and protein level (Fig. 3d, e). Co-IP showed that antiFlag antibody co-immunoprecipitated with TNF- $\alpha$, indicating that KLF14 might interact with TNF- $\alpha$ (Fig. 3f).

\section{Protection of KLF14 mediated by Treg differentiation}

Although it has previously been reported that KLF14 induces the differentiation of Tregs, in this study, we 

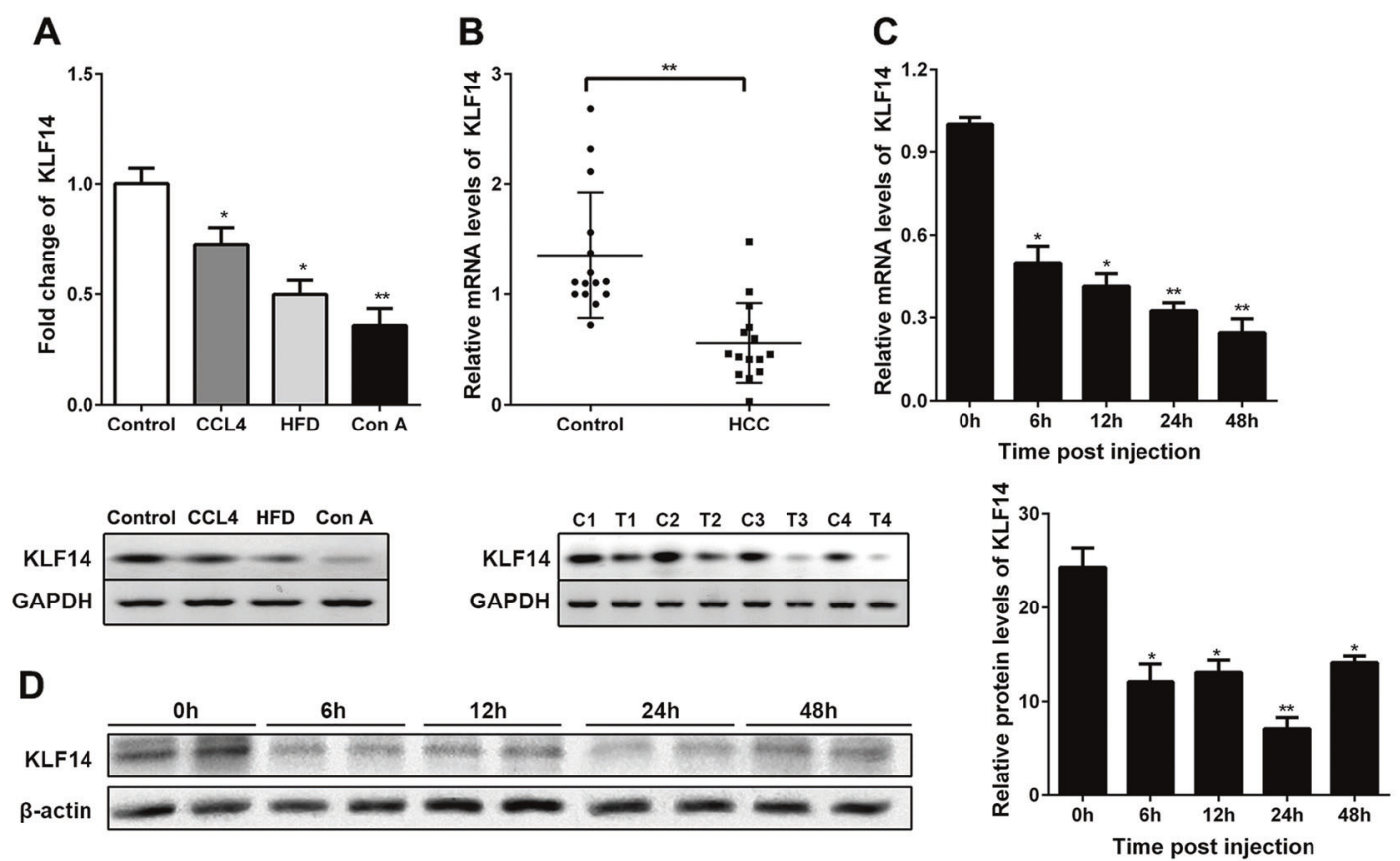

E

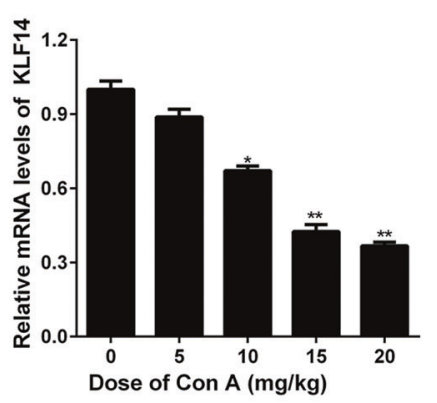

$\mathbf{F}$

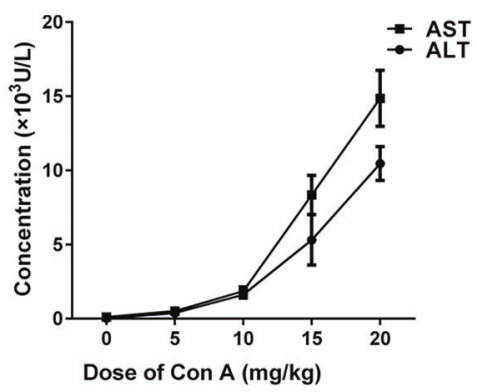

H

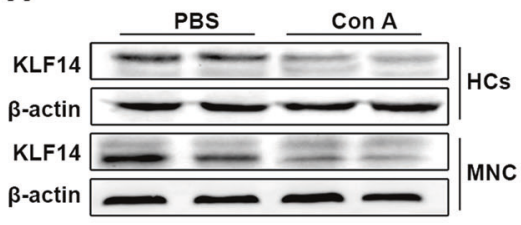

G
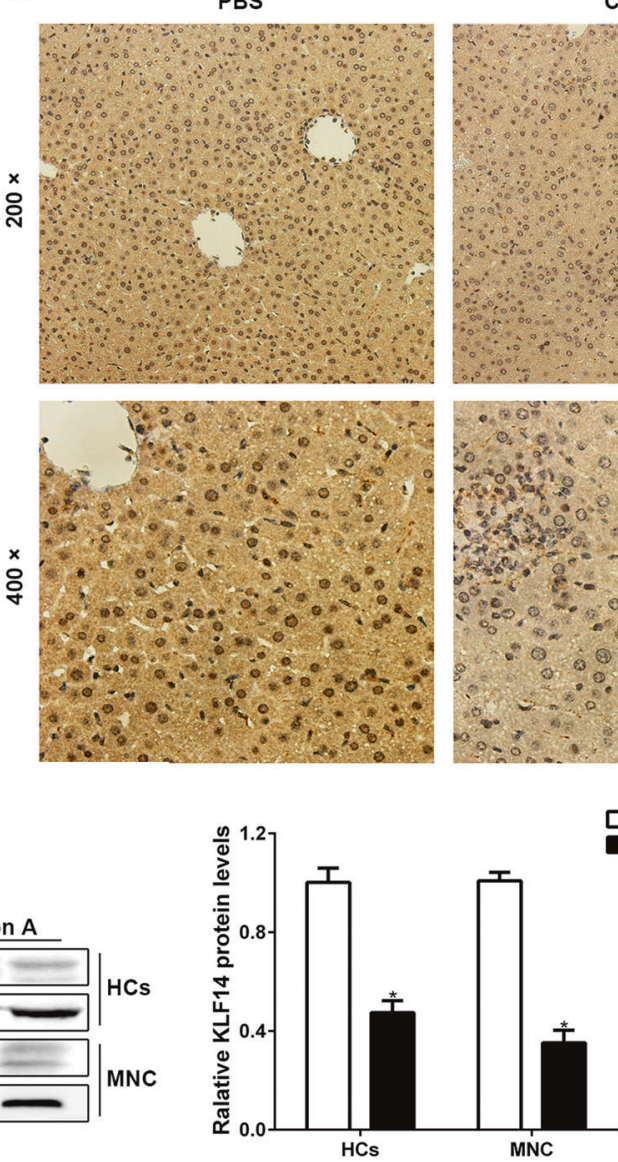

Con A
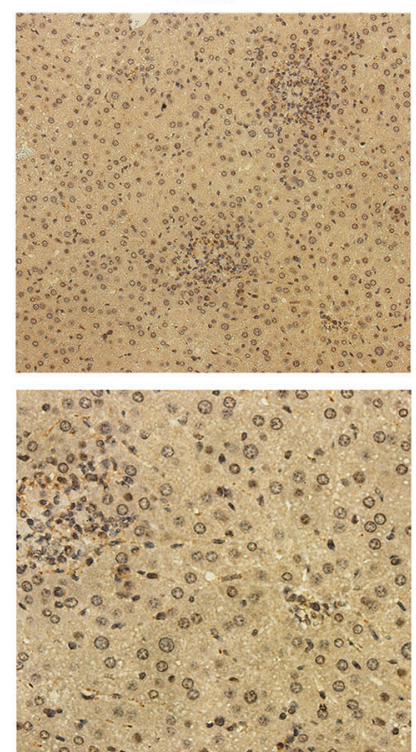

Con A

designed experiments to validate these findings. Liver MNCs and PBMCs were isolated and stained to determine the number of $\mathrm{CD} 4{ }^{+} \mathrm{CD} 25^{+} \mathrm{Foxp} 3^{+}$Tregs by using FACS.
As shown in Fig. 4a, Con A-induced immune injury significantly increased the frequency of Tregs in the liver, whereas they were decreased in blood. There was no 
Fig. 2 Downregulated KLF14 expression in chronic liver diseases. a A liver fibrosis model (CCL4, $n=8$ ) was induced by intraperitoneally injecting $20 \%$ CCL4 (at $0.5 \mu \mathrm{l} / \mathrm{g}$ of body weight twice a week) for 8 weeks. NAFLD model (HFD, $n=8$ ) was established by feeding mice with HFD (53\% of calories from fat) for 12 weeks. Immunemediated hepatitis model (Con A, $n=8$ ) was prepared with Con A as previously described. Expression of KLF14 RNA in models of various murine hepatic diseases was detected by qRT-PCR. b RNA from human liver tissue was isolated from a total of fifteen pairs of hepatocellular carcinoma (HCC) and corresponding non-tumor samples (Control). Expression of human KLF14 was measured by qRT-PCR. All these qRT-PCR results were also verified by RT-PCR (C represents HCC, $\mathrm{T}$ represents non-tumor control). Data are expressed as mean $\pm \mathrm{SD}$. $*$ and $* *$ indicated that $p<0.05$ and $p<0.01$ (vs. control), respectively. Mice $(n=5)$ were injected intravenously at a single dose of $15 \mathrm{mg} / \mathrm{kg}$ Con A. Liver samples were harvested 6, 12, 24, and $48 \mathrm{~h}$ after injection. KLF14 RNA level was detected by qRT-PCR (c) and the protein level was measured by western blotting (d). Gradient concentrations of Con A (from 5 to $20 \mathrm{mg} / \mathrm{kg}$ ) were injected into mice for $24 \mathrm{~h}(n=5)$. Expression of human KLF14 was determined by qRT-PCR (e). Meanwhile, plasma was collected for measuring the concentration of transferase level (f). Expression of KLF14 protein was immunohistochemically detected in liver tissue $(\mathbf{g})$, primary hepatocyte and MNC (h) by western blotting. ${ }^{*} p<0.05$, $* * P<0.01$

difference in the frequency of Tregs in KLF14 overexpression group and the controls. However, pretreatment with KLF14 plasmid increases the frequency of Tregs in both liver and blood. This effect was associated with its ability to induced an upregulated expression of liver MNC Foxp3 and CD25 at RNA level (Fig. 4b). To directly clarify the connection between KLF14 and Tregs, CD $4{ }^{+} \mathrm{CD} 25^{+}$ regulatory $\mathrm{T}$ cells were isolated from mice, and the expression of KLF14 was detected by qRT-PCR. As shown in Fig. 4c, Con A treatment suppressed the expression of KLF14 in Tregs. Compared with the controls (Ad-negative + Con A group), plasmid transfection (Ad-KLF14 + Con A) increased the expression of KLF14 in these cells. To determine the role of Tregs in the protective effect of KLF14, neutralizing antibodies against CD25 were injected to deplete Tregs in vivo before Con A challenge. The efficiency of this depletion ( $>98 \%$ ) was verified by FACS in the splenic Treg population (Fig. 4d). Figure 4e shows that blocking antibodies against CD25 abrogated the protective effect of KLF14 plasmids. This result was consistent with the elevated level of plasma transferase (Fig. 4f). The suppression of the inflammatory cytokines, i.e., TNF- $\alpha$ and IL6, was reversed entirely by antibodies against CD25 (Fig. 4g).

\section{Discussion}

In this study, we examined the role of KLF14 in Con Ainduced hepatitis. Our results indicate that overexpression of KLF14 alleviated the inflammatory reaction elicited by Con A. This effect was consistent with its ability to induce
Treg differentiation and to suppress the expression of inflammatory cytokines. These data suggest that KLF14 plays a role in immune-mediated hepatitis.

$\mathrm{AIH}$ is a relatively rare and heterogeneous liver disease, with a vast spectrum of clinical manifestations. Treg differentiation is believed to play an essential role in such immune-mediated conditions. A Con A-induced model affords a useful approach to selectively target $\mathrm{CD}^{+} \mathrm{T}$ cell functions in the liver disease.

KLF14 was initially identified as a ubiquitous tissueexpressed transcription factor by Scohy et al. [19]. Genomewide association studies (GWAS) indicated that it was associated with the susceptibility to lipid metabolism disorders and type 2 diabetes [20-22]. Nevertheless, several studies found that KLF14 expression was undetectable in adult murine liver (its expression was detectable in the fetal liver instead), and it plays few roles in the development of diet-induced insulin resistance in mice [23]. The weak and non-specific expression of KLF14 in all kinds of liver cells in our experiments was coincident with the findings by Scohy et al. in 2000 [19]. The reason for such discrepancies in mice liver tissue remain poorly understood. Some clues might be found in work by Utami et al. [24], who demonstrated that the KLF14 protein was a short-lived protein and rapidly degraded through a proteasome pathway. This inherent instability might be related to the unique structural proline-rich sequence at the N-terminal of the KLF14 protein, and warrants further investigation.

Although the role of KLF14 has been well-established in murine NAFLD models, the role of KLF14 overexpression in immune-mediated hepatitis warrants further study. Sarmento et al. reported that deletion of KLF14 resulted in an abnormal increase in Foxp3 at the intracellular level and an enhanced suppressive capacity of Tregs in KLF14-knockout mice [15]. However, this study yielded conflicting findings as to the role of KLF14. First, our results were in line with the previous outcome that Treg frequency was increased in the inflammatory liver in autoimmune diseases. Second, we again found that KLF14 overexpression failed to induce any significant change of Tregs, but the frequency of Tregs was increased after the mice transfected with the plasmid were challenged with Con A. The differentiation of Tregs was associated with the induced expression of CD25 and Foxp3 in liver MNC. Finally, to eliminate the casual association between KLF14 overexpression and Tregs in Con Ainduced hepatitis, mice were pretreated with neutralizing antibody to deplete Tregs in vivo. This blocking antibody abrogated the protection of KLF14 and its suppression on the inflammatory cytokines. On the basis of preliminary findings, we could only speculatively suggest that the expression and the role of KLF14 as an immediate-early gene was cell-or organ-specific, and might vary with different circumstances and stimulations. 
A
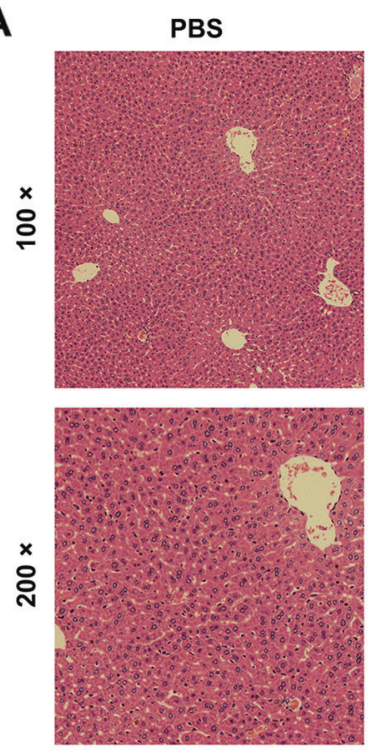

B

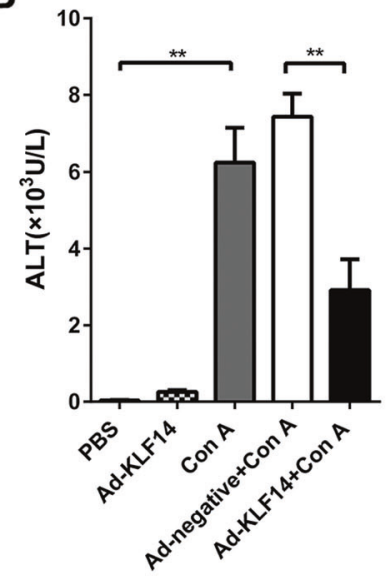

D

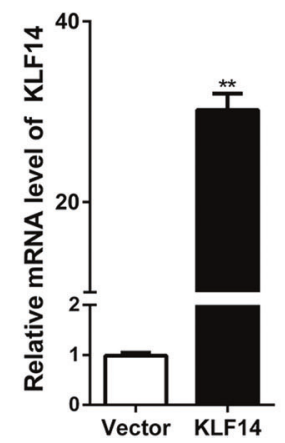

E
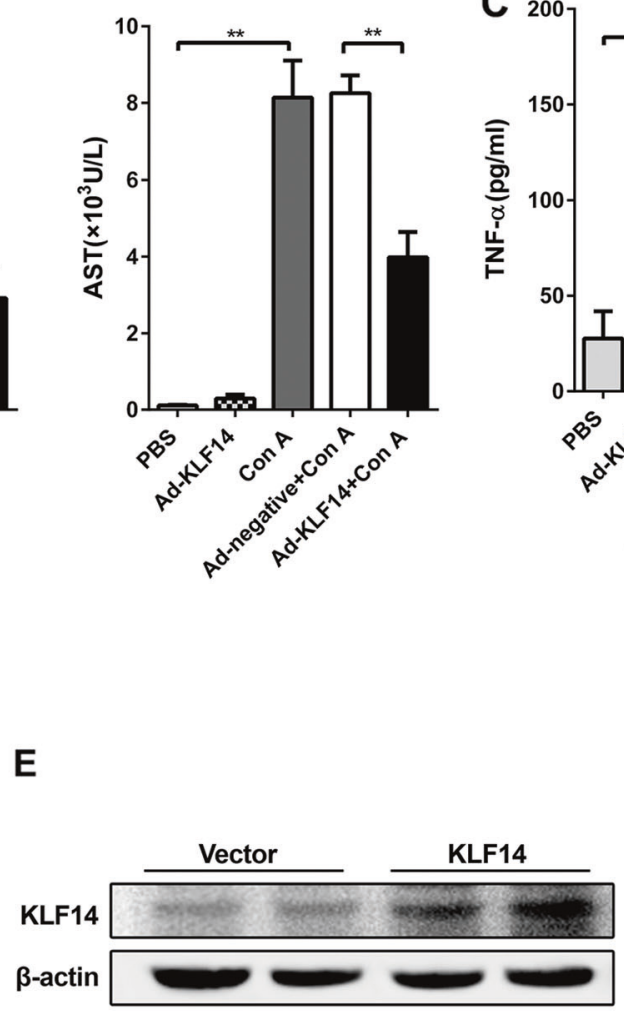

Ad-negative+Con A

Ad-KLF14+Con A
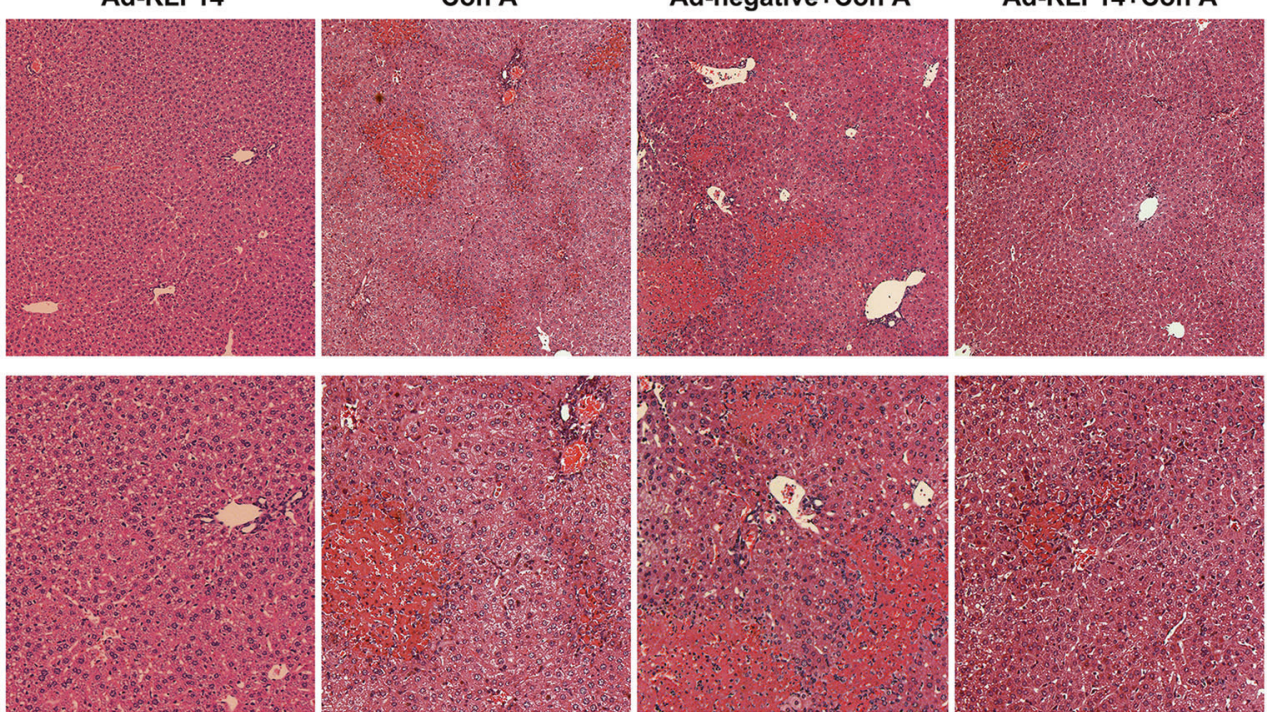
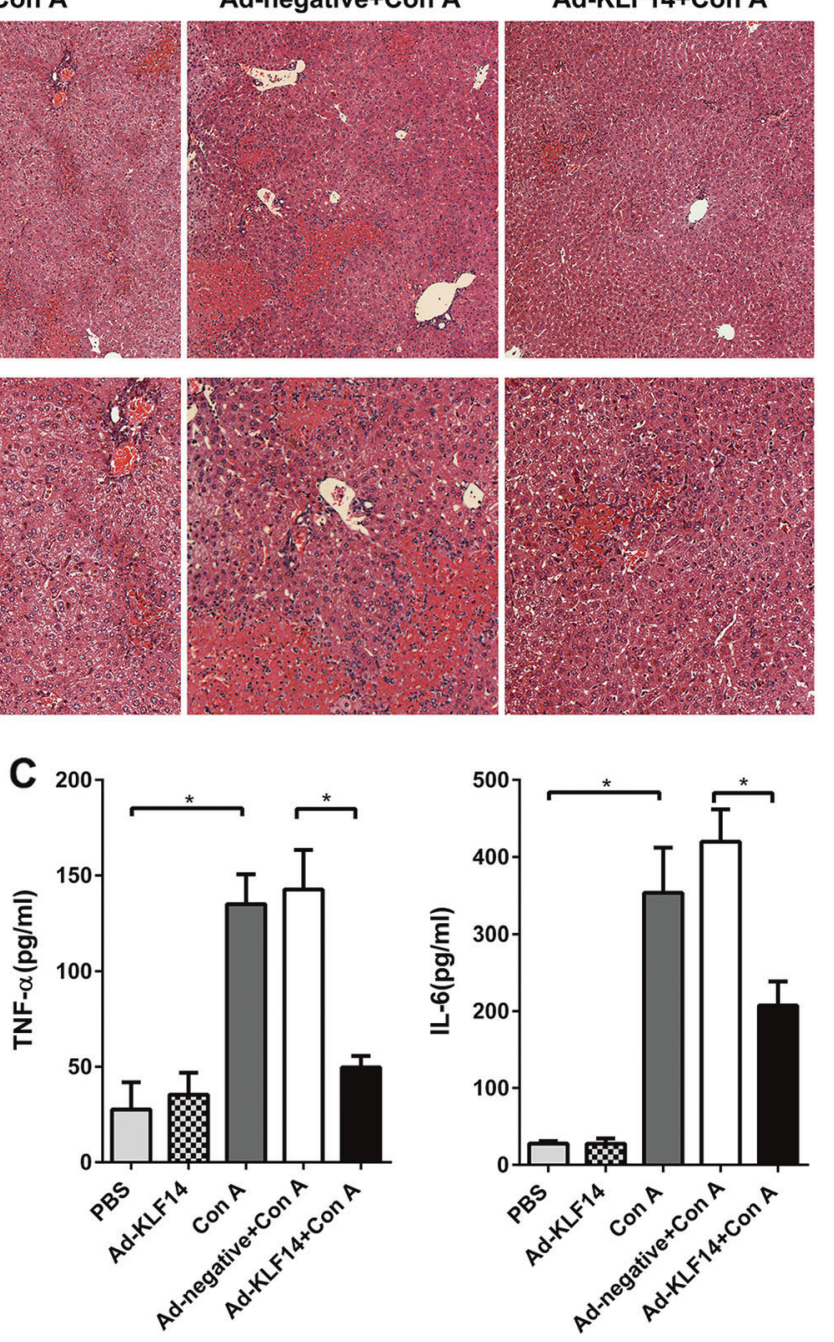

$\mathbf{F}$

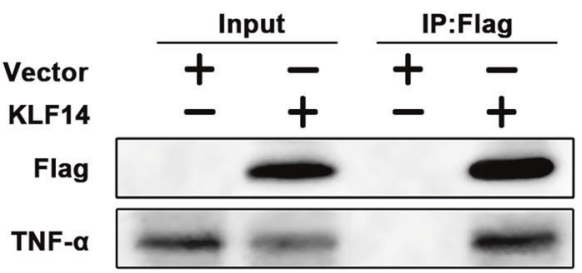

Fig. 3 Protective Effect of KLF14 overexpression on immunemediated damage. a Recombinant adenoviruses plasmid (AdKLF14) was constructed and injected into mice. After $72 \mathrm{~h}$, mice were challenged with Con A ( $15 \mathrm{mg} / \mathrm{kg})$. Liver samples were histologically HE-stained (upper, magnification $\times 100$; lower, magnification $\times 400$ ). Null type 5 adenovirus was injected as a negative control (Ad-negative). b Serum was collected from each group and tested for

A question will naturally present itself as to why the accumulating liver-infiltrating Tregs fail to hinder the disease progression. The possible explanations might be that aminotransferase level. $\mathbf{c}$ The expression of TNF- $\alpha$ and IL- 6 at protein level was also detected by ELISA. ${ }^{*} p<0.05,{ }^{* *} p<0.01$ vs. PBS or Adnegative + Con A. RAW264.7 cells were transfected with plasmids encoding Flag-KLF14. Overexpression of KLF14 was confirmed by qRT-PCR (d) and western blotting (e). After $72 \mathrm{~h}$, cell lysates were prepared and used for co-immunoprecipitation (f)

the imbalance between overly strong inflammatory stimuli and the suppression of the activity of intrahepatic Tregs. Moreover, the plasticity of Tregs themselves might inhibit 
A
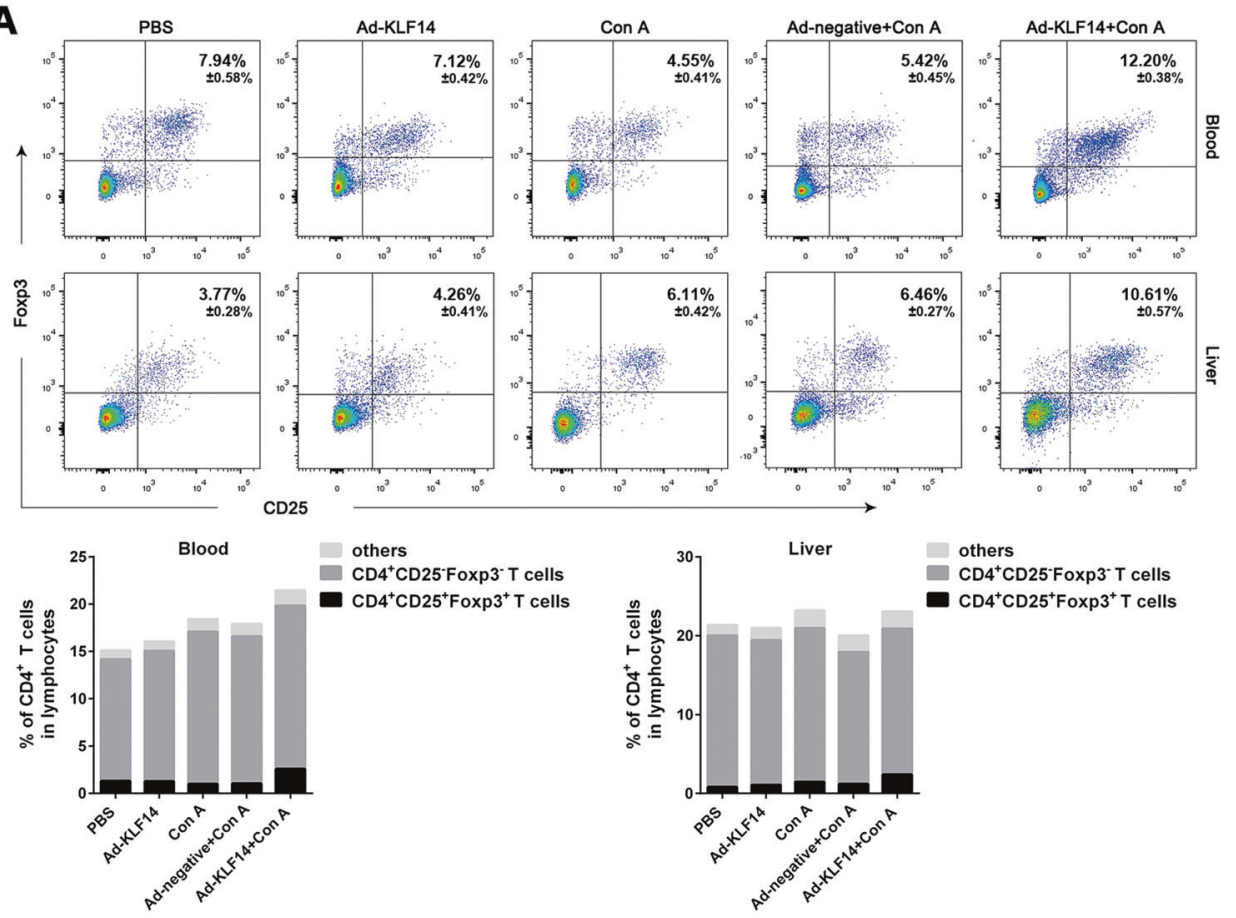

B

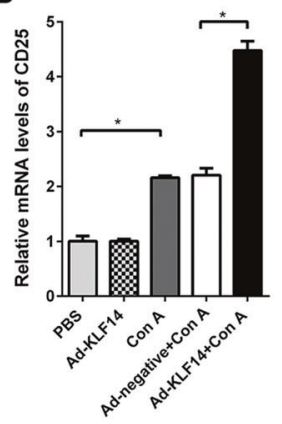

E
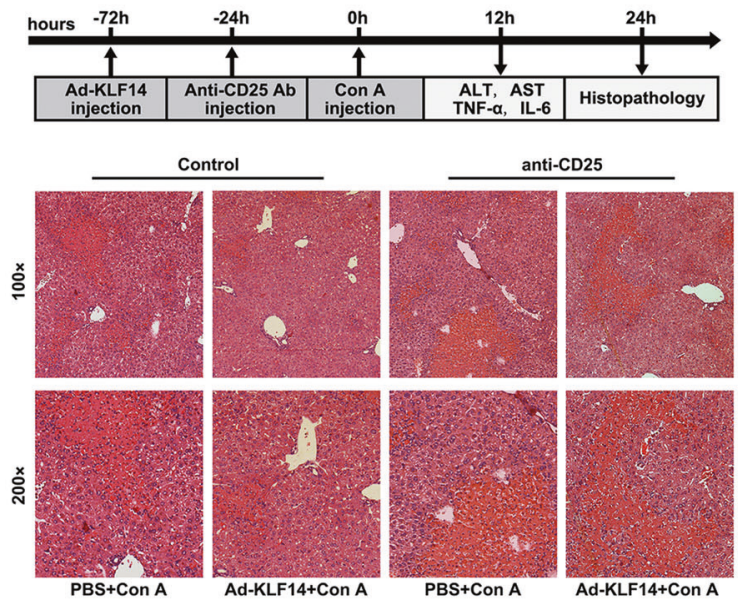

their suppressive function [25]. Given the contradictory data, which were all based on the same KLF14 knockout

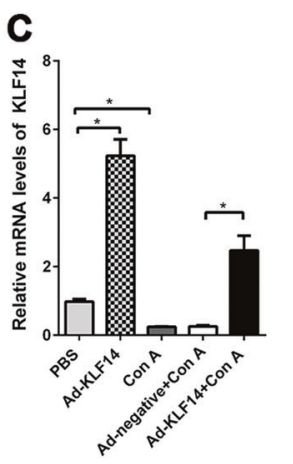

D
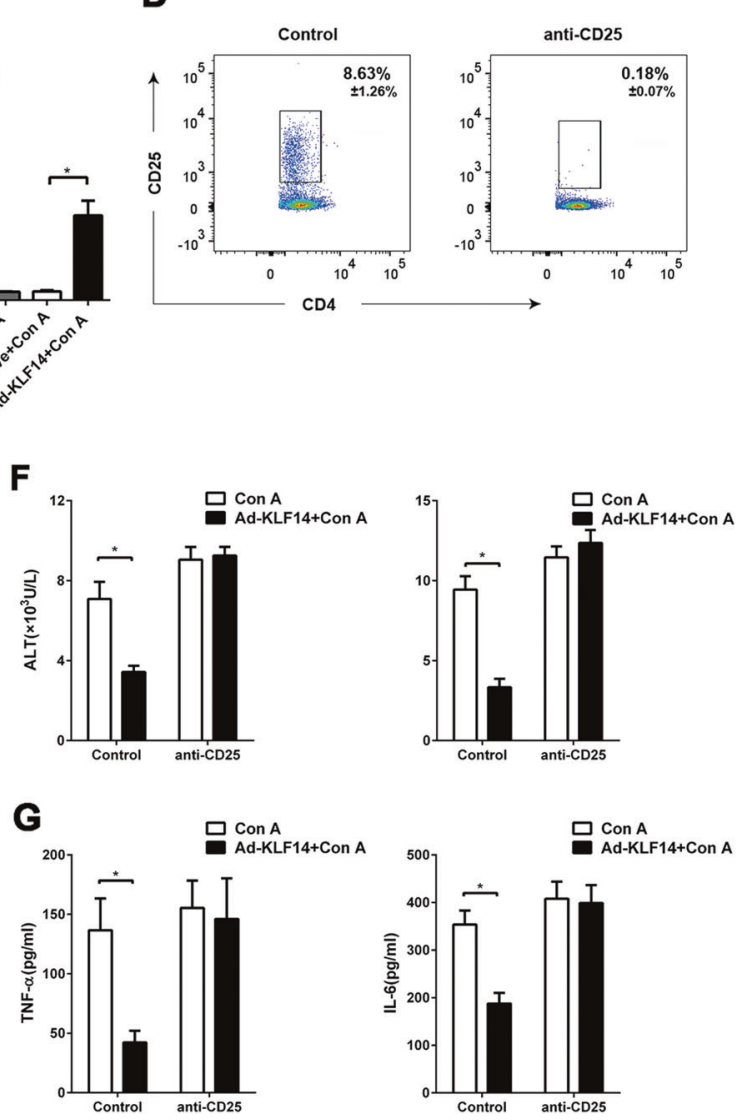

mice models [15, 23], the plasticity of Tregs with diverse etiology needs further research. 
Fig. 4 Protection of KLF14 mediated by Treg differentiation. a Recombinant adenoviruses plasmid (Ad-KLF14) was injected into mice. After $72 \mathrm{~h}$, mice were subjected to challenge with Con A (15 mg/ $\mathrm{kg}$ ). Liver MNCs were isolated from each group for FACS detection of Tregs. Cells were gated on viable lymphocytes by their light-scattering feature. Percentages (mean $\pm \mathrm{SD}$ ) of $\mathrm{CD} 4^{+} \mathrm{CD} 25^{+} \mathrm{Foxp}^{+}$Tregs within the $\mathrm{CD} 4^{+} \mathrm{T}$ cell population were labeled in the figure (upper) and demonstrated with the column graph (lower). b Expression of Foxp3 and CD25 in Liver MNC was detected by qRT-PCR. ${ }^{*} p<0.05$ vs. PBS or Ad-negative + Con A. c CD $4{ }^{+} \mathrm{CD} 25^{+}$regulatory T cells were prepared by magnetic cell sorting. Expression of KLF14 was detected by qRT-PCR. $\mathbf{d}$ In vivo depletion of $\mathrm{CD} 4{ }^{+} \mathrm{CD} 25^{+}$Tregs was achieved by intraperitoneal injection of anti-CD25 neutralizing antibody. Splenocytes were then purified to verify the depletion efficiency by FACS regarding the percentage of $\mathrm{CD} 25^{+}$Tregs. e Mice transfected with Ad-KLF14 were injected with anti-CD25 neutralizing antibody after $48 \mathrm{~h}$. After $24 \mathrm{~h}$, these mice were treated with Con A $(15 \mathrm{mg} / \mathrm{kg}) 24 \mathrm{~h}$. Liver injury was immunohistochemically detected. Serum transferase (f) and the expression of TNF- $\alpha$ and IL-6 protein (g) were also determined. ${ }^{*} p<0.05$ Con A vs. Ad-KLF14 + Con A

It has been widely recognized that $\mathrm{AIH}$ is a condition mediated principally by $\mathrm{T}$ cells. Moreover, inflammatory cytokines, such as TNF- $\alpha$ and IL-6 play the critical roles in the pathogenesis of the disease. These two factors were shown to be significantly higher in AIH patients [26], which was further verified in our study. We showed that the changes in the cytokines were associated with the severity of the hepatic injury. We exhibited KLF14 and TNF- $\alpha$ coimmunoprecipitated in macrophage cell line suggesting that they are functionally related. A small case-control study described the possible effect of infliximab on steroidsinsensitive patients [27]. Our results afforded at least one probability that the KLF14 might serve as an agonist or adjuvant agent in the treatment of AIH.

Furthermore, we also found that blocking Treg differentiation could completely reverse the suppressive action of inflammatory cytokines induced by KLF14 overexpression. It has been reported that TNF- $\alpha$ impaired liver function in $\mathrm{T}$ cells-mediated autoimmune disease patients [28]. However, few studies examined the direct effect of Tregs on inflammatory cytokines in AIH. It is well-known that, compared to other $\mathrm{T}$ cells, Tregs express high levels of TNF- $\alpha$ receptor 2 (TNFR2) [29], but its mechanism will be determined in further studies.

In conclusion, our data show that KLF14 overexpression protected mouse liver from Con A-induced immune damage. KLF14 might work via two pathways, i.e., Treg differentiation and inflammatory cytokine suppression. Our findings expanded the knowledge about the role of the KLF14 and provided a possibility to use KLF14 as a target for the treatment of AIH.

Acknowledgements DT is supported by the National Natural Science Foundation of China (81572419).

\section{Compliance with ethical standards}

Conflict of interest The authors declare that they have no conflict of interest.

\section{References}

1. Czaja AJ, Manns MP. Advances in the diagnosis, pathogenesis, and management of autoimmune hepatitis. Gastroenterology. 2010;139:58-72.

2. Sebode M, Schramm C. AIH: which alternative for difficult-totreat patients? Dig Dis. 2015;33:83-87.

3. Liberal R, Krawitt EL, Vierling JM, Manns MP, Mieli-Vergani G, Vergani D. Cutting edge issues in autoimmune hepatitis. J Autoimmun. 2016;75:6-19.

4. Krawitt EL. Autoimmune hepatitis. N Engl J Med. 2006;354:54-66.

5. Liberal R, Longhi MS, Mieli-Vergani G, Vergani D. Pathogenesis of autoimmune hepatitis. Best Pract Res Clin Gastroenterol. 2011;25:653-64.

6. Longhi MS, Hussain MJ, Mitry RR, Arora SK, Mieli-Vergani G, Vergani D, et al. Functional study of CD4+CD25+regulatory $\mathrm{T}$ cells in health and autoimmune hepatitis. J Immunol. 2006;176:4484-91.

7. Peiseler M, Sebode M, Franke B, Wortmann F, Schwinge D, Quaas A, et al. FOXP3+regulatory T cells in autoimmune hepatitis are fully functional and not reduced in frequency. J Hepatol. 2012;57:125-32.

8. Heymann F, Hamesch K, Weiskirchen R, Tacke F. The concanavalin A model of acute hepatitis in mice. Lab Anim. 2015;49:12-20.

9. Mangano K, Cavalli E, Mammana S, Basile MS, Caltabiano R, Pesce A, et al. Involvement of the Nrf2/HO-1/CO axis and therapeutic intervention with the CO-releasing molecule CORM-A1, in a murine model of autoimmune hepatitis. J Cell Physiol. 2018;233:4156-65.

10. Presnell JS, Schnitzler CE, Browne WE. KLF/SP transcription factor family evolution: expansion, diversification, and innovation in eukaryotes. Genome Biol Evol. 2015;7:2289-309.

11. Kim CK, He P, Bialkowska AB, Yang VW. SP and KLF transcription factors in digestive physiology and diseases. Gastroenterology. 2017;152:1845-75.

12. Cao Z, Sun X, Icli B, Wara AK, Feinberg MW. Role of Krüppellike factors in leukocyte development, function, and disease. Blood. 2010;116:4404-14.

13. Pabbisetty SK, Rabacal W, Volanakis EJ, Parekh VV, OlivaresVillagómez D, Cendron D, et al. Peripheral tolerance can be modified by altering KLF2-regulated Treg migration. Proc Natl Acad Sci USA. 2016;113:E4662-4670.

14. Khedkar SA, Sun X, Rigby AC, Feinberg MW. Discovery of small molecule inhibitors to Krüppel-like factor 10 (KLF10): implications for modulation of $\mathrm{T}$ regulatory cell differentiation. $\mathrm{J}$ Med Chem. 2015;58:1466-78.

15. Sarmento OF, Svingen PA, Xiong Y, Xavier RJ, McGovern D, Smyrk TC, et al. A novel role for KLF14 in T regulatory cell differentiation. Cell Mol Gastroenterol Hepatol. 2015;1:188-202.

16. Vasseur P, Serres L, Jégou JF, Pohin M, Delwail A, Petit-Paris I, et al. High-fat diet-induced IL-17A exacerbates psoriasiform dermatitis in a mouse model of Steatohepatitis. Am J Pathol. 2016;186:2292-301.

17. King A, Houlihan DD, Kavanagh D, Haldar D, Luu N, Owen A, et al. Sphingosine-1-phosphate prevents egress of hematopoietic stem cells from liver to reduce fibrosis. Gastroenterology. 2017;153:233-48. 
18. Diao W, Jin F, Wang B, Zhang CY, Chen J, Zen K, et al. The protective role of myeloid-derived suppressor cells in concanavalin A-induced hepatic injury. Protein Cell. 2014;5:714-24.

19. Scohy S, Gabant P, Van Reeth T, Hertveldt V, Drèze PL, Van Vooren P, et al. Identification of KLF13 and KLF14 (SP6), novel members of the SP/XKLF transcription factor family. Genomics. 2000;70:93-101.

20. Xie W, Li L, Zheng XL, Yin WD, Tang CK. The role of Krüppellike factor 14 in the pathogenesis of atherosclerosis. Atherosclerosis. 2017;263:352-60.

21. Guo Y, Fan Y, Zhang J, Lomberk GA, Zhou Z, Sun L, et al. Perhexiline activates KLF14 and reduces atherosclerosis by modulating ApoA-I production. J Clin Invest. 2015;125:3819-30.

22. Yang M, Ren Y, Lin Z, Tang C, Jia Y, Lai Y, et al. Krüppel-like factor 14 increases insulin sensitivity through activation of PI3K/ Akt signal pathway. Cell Signal. 2015;27:2201-8.

23. Argmann CA, Violante S, Dodatko T, Amaro MP, Hagen J, Gillespie VL, et al. Germline deletion of Krüppel-like factor 14 does not increase risk of diet induced metabolic syndrome in male C57BL/6 mice. Biochim Biophys Acta. 2017;1863:3277-85.
24. Utami TW, Miyoshi K, Hagita H, Yanuaryska RD, Horiguchi T, Noma T. Possible linkage of SP6 transcriptional activity with amelogenesis by protein stabilization. J Biomed Biotechnol. 2011;2011:320987.

25. Behairy BE, El-Araby HA, Abd El Kader HH, Ehsan NA, Salem ME, Zakaria HM, et al. Assessment of intrahepatic regulatory $\mathrm{T}$ cells in children with autoimmune hepatitis. Ann Hepatol. 2016;15:682-90.

26. Akberova D, Kiassov AP, Abdulganieva D. Serum cytokine levels and their relation to clinical features in patients with autoimmune liver diseases. J Immunol Res. 2017;2017:9829436.

27. Weiler-Normann C, Schramm C, Quaas A, Wiegard C, Glaubke $\mathrm{C}$, Pannicke N, et al. Infliximab as a rescue treatment in difficultto-treat autoimmune hepatitis. J Hepatol. 2013;58:529-34.

28. Bo X, Broome U, Remberger M, Sumitran-Holgersson S. Tumour necrosis factor alpha impairs function of liver derived $\mathrm{T}$ lymphocytes and natural killer cells in patients with primary sclerosing cholangitis. Gut. 2001;49:131-41.

29. Cohen JL, Wood KJ. TNFR2: the new Treg switch? Oncoimmunology. 2017;7:e1373236. 